\title{
Space charge behaviour in oil and impregnated pressboard combined insulation system
}

\author{
Miao Hao*, Yuan Zhou, George Chen \\ University of Southampton Southampton, \\ United Kingdom \\ *mh2e10@soton.ac.uk
}

\begin{abstract}
Dielectric performance of oil-pressboard insulation system used in high voltage direct current (HVDC) convertor transformers can be affected by the presence of space charge. In this paper, the space charge behaviors in a $0.5 \mathrm{~mm}$ thick oil film combined with a $1 \mathrm{~mm}$ thick impregnated pressboard have been investigated by the pulsed electroacoustic (PEA) technique under $12 \mathrm{kV} / \mathrm{mm}$ and $20 \mathrm{kV} / \mathrm{mm}$ at room temperature. Two types of oil with different aging status were used for comparison. The results show that a charge peak is quickly formed at the interface between oil and pressboard with the same polarity as the electrode at the oil film side. However, the dynamics of the interfacial charges are very different for fresh oil and aged oil samples. The maximum electric field occurs in the middle of the pressboard, which is significantly enhanced in the aged oil samples. Difference in the electric field between those obtained from measurement results and those calculated from Maxwell- Wagner theory has been discussed.
\end{abstract}

Keywords—space charge, oil pressboard, interface, aging, PEA, HVDC.

\section{INTRODUCTION}

With a rapid development in HVDC technique, researches on the insulation material, design, test and manufactory applied in converter transformers have attracted more attention. As the main insulation in converter transformer, oilpaper/pressboard insulation system usually suffers from space charge phenomena under HVDC voltages. The presence of space charge can distort the local electric field distribution in the dielectric material, leading to accelerated aging or even failure [1]. Most published works were concentrated on either thin impregnated paper [2-4] or multi-layer papers [5,6]. The results indicate that space charge dynamics are highly dependent on the applied voltage, environmental condition, and sample status. Moreover, the interface between two layers of paper can prevent charges passing through and also reduce the charge mobility [7]. However, in a real converter transformer, the pressboards are extensively used Therefore, the knowledge on the interface between oil and pressboard are highly desired since the interface is generally believed as the weakest point in an insulation system [8].

In this paper, the space charge behaviours in the oil film combined with oil impregnated pressboard are investigated by the means of PEA technique. The work focuses on the status of oil and its influence on space charge behaviours at the interfaces. The impact of these interfacial space charges on electric field enhancement in the oil/pressboard insulation system has been discussed.

\author{
Gordon Wilson, Paul Jarman \\ National Grid plc. \\ United Kingdom
}

\section{EXPERIMENTAL SETUP}

In order to investigate the impacts of aging of the transformer oil on the space charge dynamics, two types of oil were used in this study: fresh oil and serviced aged oil. The fresh oil has high oxidation stability and low sulphur content to meet IEC 60296, while the aged oil, supplied by National Grid, was collected from a long term serviced power transformer. Table 1 describes the status of the two different types of oil. The appearance and the results of UV/Vis spectra, shown in Fig. 1, clearly indicate the significant difference.

Table 1. Status of the oil properties at $20^{\circ} \mathrm{C}$

\begin{tabular}{c|c|c}
\hline & Fresh oil & Aged oil \\
\hline Moisture $(\mathrm{ppm})$ & 10 & 25 \\
Resistivity $(\mathrm{T} \Omega \mathrm{m})$ & 7 & 0.1 \\
Relative Permittivity & 2.2 & 2.6 \\
Description & transparent & dark brown \\
& & with sludge \\
\hline
\end{tabular}
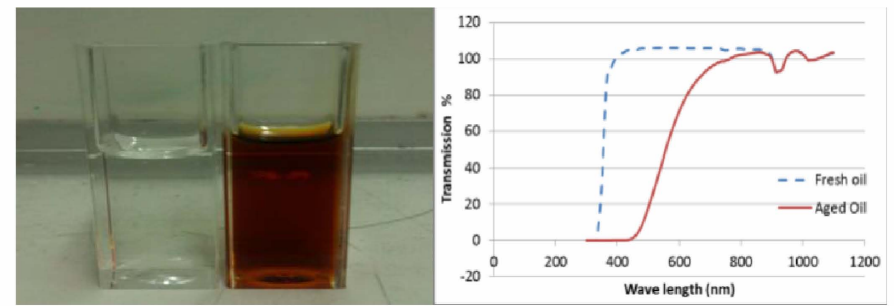

Fig. 1. The color difference and the UV/Vis spectra results

The pressboard used in this work was the normal insulating pressboard with $1 \mathrm{~mm}$ in thickness and pressed pattern surface. The pressboard samples were cut into circular shape with a diameter of $9 \mathrm{~cm}$ to fit the electrode and avoid flashover. The pressboards were dried at $80^{\circ} \mathrm{C}$ for 3 days in a vacuum oven. After that, the dried pressboards were separately impregnated in the two types of oil under $10 \mathrm{~Pa} / 60$ ${ }^{\circ} \mathrm{C}$ for 3 days, to ensure the pressboards were fully impregnated with oil. The unused samples were kept in a seal container under vacuum condition to avoid absorbing moisture and possible contamination. The dielectric properties of the impregnated pressboards are shown in Table 2.

Table 2. Dielectric properties of impregnated pressboards

\begin{tabular}{|c|c|c|}
\hline & Fresh oil-PB & Aged oil-PB \\
\hline $\begin{array}{c}\text { Resistivity }(\mathrm{T} \Omega \mathrm{m}) \\
\text { Relative Permittivity }\end{array}$ & $\begin{array}{l}140 \\
3.2\end{array}$ & $\begin{array}{l}0.3 \\
4.2\end{array}$ \\
\hline
\end{tabular}


To provide an oil gap (film), the aluminum plate (ground electrode of the PEA system) was modified to an oil container with a $5 \mathrm{~mm}$ in depth and $10 \mathrm{~cm}$ in diameter, as shown in Fig. 2 (A).

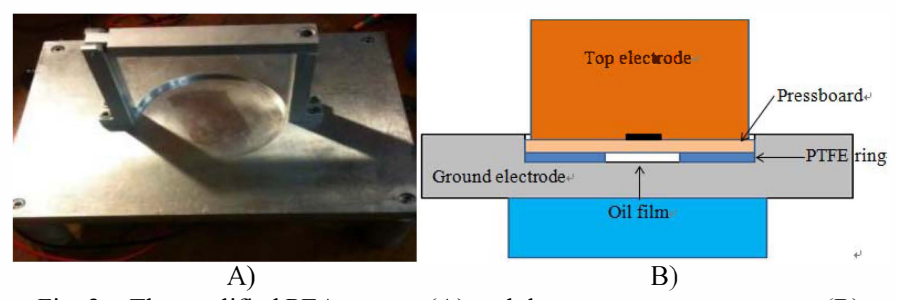

Fig. 2. The modified PEA system (A) and the measurement structure (B).

To achieve a firm contact between the sample and electrodes (essential for acoustic signal transmission) and an accurate thickness of oil film, a $0.5 \mathrm{~mm}$ thick PTFE ring was placed on the aluminium electrode. The pressboard was firmly pressed by the top electrode as shown in Fig. 2 (B).

All the measurements were carried out at the room temperature. Two DC electric fields were applied, $12 \mathrm{kV} / \mathrm{mm}$ and $20 \mathrm{kV} / \mathrm{mm}$, as comparison. The volts-on tests were carried out during the voltage application period ( 3 hours). Then the voltage was turned off, and the decay tests started.

\section{EXPERIMENT RESULTS}

\section{A. Fresh oil sample}

\section{1) Volts-on}

The volts-on results for the fresh oil and fresh oilimpregnated pressboard under $12 \mathrm{kV} / \mathrm{mm}$ (A) and $20 \mathrm{kV} / \mathrm{mm}$ (B) are shown in Fig. 3.

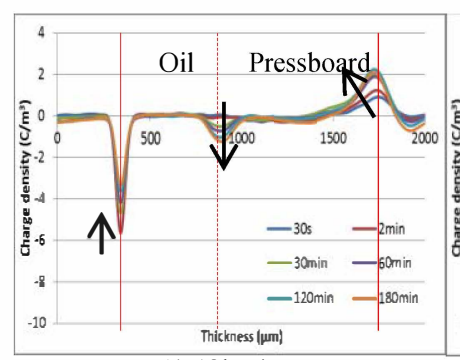

A) $12 \mathrm{kV} / \mathrm{mm}$

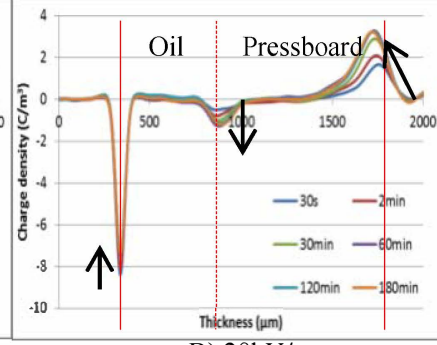

B) $20 \mathrm{kV} / \mathrm{mm}$
Fig. 3. Volts-on results of fresh oil samples under different electric fields.

A peak is formed at the interface between oil and pressboard. Its polarity is the same as the polarity of the oil side electrode (negative in this case). This agrees with the Maxwell-Wagner polarization [9] that charges will be formed at the interface when the dielectrics have discontinuity of the ratio of permittivity and conductivity. Most of the interfacial charges are negative since $\left[\sigma_{p} / \sigma_{\sigma}\right) \sigma_{n}\left(s_{p} / a_{\sigma}\right)$, where the $\sigma_{\mathrm{p}}$ and $\sigma_{0}$ represent the conductivity of impregnated pressboard and oil; the $\varepsilon_{\mathrm{p}}$ and $\varepsilon_{\mathrm{o}}$ represent the permittivity of the impregnated pressboard and oil.

At the oil side, the cathode peak decreases with the time, which indicates homo charge injection occurs in the oil film. The injected negative charges move into the insulation system, and their movement is hindered by the interface. Therefore, large amount of negative charges start to accumulate at the interface, forming an increasing negative peak.

At the same time, positive charges are injected from the anode side. It is observed that the anode peak moves into the bulk of the pressboard with an increasing amplitude. This is because the anode peak detected by the PEA system is the combination of the injected homo charges and the induced charges on the electrode from the interfacial charge in the middle. Moreover, these charges are difficult to be distinguished due to the large acoustic attenuation and dissipation on the anode side. Fig.4 shows a schematic representation of this phenomenon. The blue charges indicate injected charges, and the red ones indicate the induced charges.
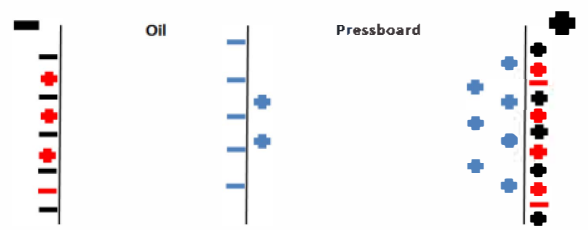

Fig. 4. The illustration of net charges detected by the PEA technique

The time of the interfacial peak build-ups in the samples under different voltages is different, i.e. a considerable large negative peak formed at the interface within the first 30 s under $20 \mathrm{kV} / \mathrm{mm}$, while there is no such obvious peak under $12 \mathrm{kV} / \mathrm{mm}$ at the same time. This could be explained by the Maxwell theory as the charges build up faster under higher electric field if the time constant is similar. Moreover, the conductivity of the dielectric is usually field dependent, which can also affect the interfacial charges behaviour.

\section{2) Decay}

After the 3 hours volts-on test, we turned off the external electric field to start the decay test. Similarly, there is 3 hours for the decay test which usually can show the movement of the injected charges without the impacts of the external electric field. Therefore, the decay process can provide more information on the characteristics of the dielectrics themselves.

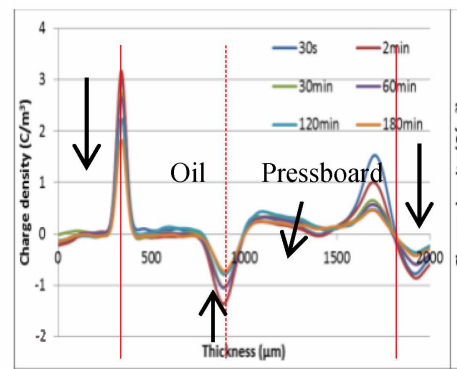

A) $12 \mathrm{kV} / \mathrm{mm}$

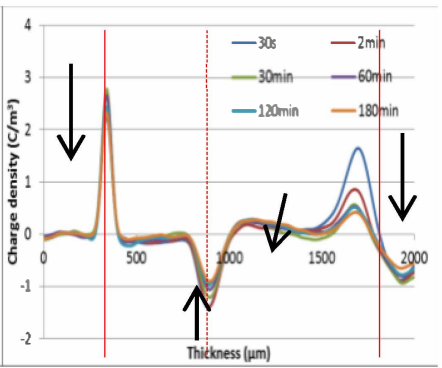

B) $20 \mathrm{kV} / \mathrm{mm}$
Fig. 5. Charge decay in fresh oil samples after the applied voltage removal.

The decay results of the fresh oil samples under $12 \mathrm{kV} / \mathrm{mm}$ (A) and $20 \mathrm{kV} / \mathrm{mm}$ (B) are shown in Fig. 5. The positive peak at the cathode (left) is induced by the injected negative charges during the volts-on process. The injected charges decay with time, so does the induced peak. At the interface, one can see a large amount of negative charges accumulated during the volts-on process. Moreover, there is a positive peak that can be observed on the pressboard side of the interface. Considering only the net charge can be measured by the PEA 
method, this positive peak can be explained as combination of the accumulated positive and negative charges near the interface area as shown in Fig. 4. Therefore, the interface seems to act as a barrier and can prevent the movement of both polarity of charges, which agrees with the previous research finding in [5] and [11].

On the anode side, the injected positive charges decrease fast in the first 2 mins and then slow down. Due to the fact of significant attenuation and dissipation in thick pressboard, the injected positive charges are overlapped with the induced charges at the anode, resulting in a big positive peak observed in this case.

\section{B. Aged oil sample}

\section{1) Volts-on}

The space charge profiles for aged oil samples are shown in Fig. 6 under $12 \mathrm{kV} / \mathrm{mm}$ (A) and $20 \mathrm{kV} / \mathrm{mm}$ (B). They are very different compared with those in Fig. 3 for fresh oil samples.

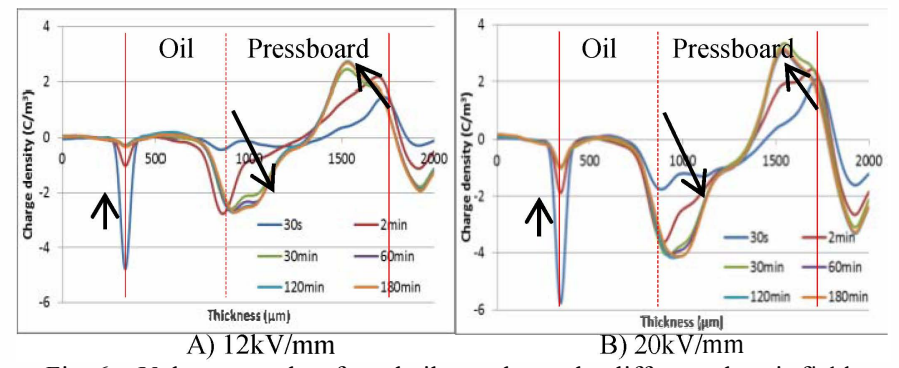

Fig. 6. Volts-on results of aged oil samples under different electric fields.

Generally, the space charge movement is much more significant in the aged oil samples than fresh ones. The cathode peak decreases very quickly and almost disappears after 2 mins which indicates very strong homo charge injection. A large amount of negative charges move to the interface and quickly form a significant negative peak at the interface. Moreover, the negative charges intend to move into the pressboard after 5 mins. In this case, the interface looks like a new cathode. Similarly, strong homo charge injection also happens at the anode, the positive peak moves into the insulation bulk. Consequently, the middle region of the pressboard endures a very high electric field. The higher the applied electric field, the more the injected charge as shown in Fig. 6 (B), the higher the electric filed in the pressboard.

\section{2) Decay}

After 3 hours, the applied voltage is removed and the decay test starts. Only the results in the first 5mins are recorded, because the movement of charges is very fast in the aged oil samples, most of the charges disappear shortly after the removal of the applied fields, as shown in Fig.7 (A) and (B). This may be related to the higher conductivity of the aged oil as shown in Table 1.

The accumulated charges in the oil can drift away very rapidly, so the induced charge peak at the cathode disappears quickly: almost no charges at all in 5 mins. On the other hand, the movement of the charges accumulated in the pressboard seems little slower and some space charge remains after 5 mins decay.

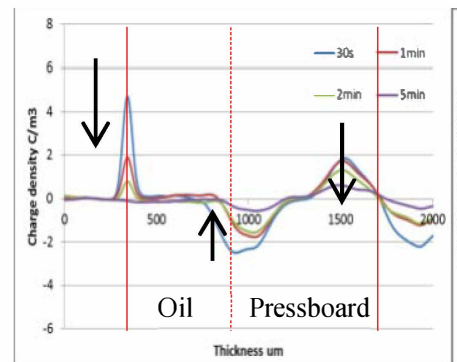

A) $12 \mathrm{kV} / \mathrm{mm}$

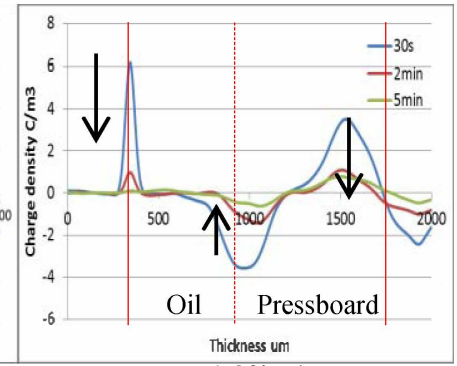

B) $20 \mathrm{kV} / \mathrm{mm}$
Fig. 7. Charge decay in aged oil samples after the applied voltage removal.

\section{ANALYSIS AND DISCUSSION}

\section{A. Total absolute charge amount}

The total absolute charge amount is usually used to provide a general comparison between different samples. The charge amount can be easily calculated using equation (1) from the charge density measured:

$$
Q(t)=\int_{0}^{d}|\rho(x, t)| S d x
$$

where $d$ is the thickness of the sample, $\rho(x, t)$ is the charge density, $\mathrm{S}$ is the area of the electrode. The results are shown in Fig. 8, the volts-on shown in A) and the decay shown in B).

According to the plot A), the amount of space charge in the samples increases quickly in the first 10 mins and then the rate of increase slows down gradually during the voltage application. It is noted that the rate of charge build-up in the present arrangement is much quicker than that observed in oil impregnated pressboard only [10]. This demonstrates the impact of oil film in the present system on charge formation possibly due to the combination of the higher oil conductivity and the interface. Moreover, the status of the oil property domains the charge amount during the volts-on test, e.g. the charge amount in the aged oil samples under $12 \mathrm{kV} / \mathrm{mm}$ is much larger than in the fresh oil samples under $20 \mathrm{kV} / \mathrm{mm}$.

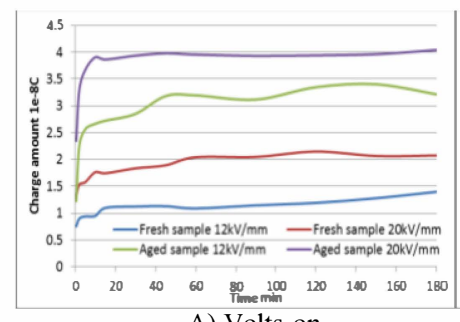

A) Volts-on

Fig. 8. The total absolute charge amount.

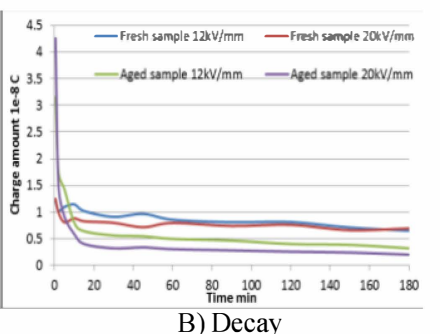

B) Decay
The charge amount during the depolarization process is shown in the plot B). The amount of the accumulated charges decreases rapidly in the first 5 mins in the aged oil samples (from $4.43 * 10^{-8} \mathrm{C}$ to about $0.8 * 10^{-8} \mathrm{C}$ ), and only very little charges remain after 20 mins. On the contrary, the accumulated charges in the fresh oil samples decay quickly in the first 10 mins and then decay little in the next 170 mins. Consequently, significant amount of charges (compared with the accumulated charges) remains in the samples. 


\section{B. Electric field distortion}

Electric field distortion is one of the major concerns caused by space charge accumulation. It is important to know the electric field distribution along the sample, which can be calculated based on space charge distribution:

$$
\mathrm{E}(\mathrm{x})=\int_{0}^{\mathrm{x}} \frac{\rho(\mathrm{x})}{\varepsilon_{0} \varepsilon_{\mathrm{r}}} \mathrm{dx} \quad 0 \leq \mathrm{x} \leq \mathrm{d}
$$

where $\rho(x)$ is the charge density, $\varepsilon_{0}$ is the vacuum permittivity, $\varepsilon_{\mathrm{r}}$ is the relative permittivity of test sample, $d$ is the thickness of the sample. The calculated electric field distribution shows continuously a reduction in the oil film and an increase in the pressboard with the voltage application Particularly, in the aged oil samples, due to the significant charge injection the electric field in the middle of the pressboard is greatly enhanced.

According to the MWS polarization, the time constant that reaches to the steady state can be calculated by:

$$
\tau_{M W}=s_{0} \frac{s_{p}+s_{p}}{a_{0}+e_{p}}
$$

where the $\sigma_{p}$ and $\sigma_{o}$ represent the conductivity of impregnated pressboard and oil; the $\varepsilon_{\mathrm{p}}$ and $\varepsilon_{\mathrm{o}}$ represent the permittivity of the impregnated pressboard and oil. The simple calculation can be made, based on the dielectric properties and the equation above. The time constants for the two types of samples are 2.1 hours for fresh oil sample; 24 seconds for aged oil sample. When the steady state reached, the difference of the electric field (calculated from equation 2) distributed in the fresh oil and pressboard is about 1:2.5 which is much smaller than the conducitivty ratio between the fresh oil and pressboard (1:14). However, in the aged oil sample, the difference of the electric field distribution is about 1:1.7 while the conductivity ratio between aged oil and pressboard is 1:3. There are some possible reasons for this: firstly, the impact caused by the interface, and the moreover, the ability to block charges caused by the status of the oil can also influence the electric field distribution in the oil and pressboard. Secondly, the conductivity of the samples is electric field dependent and also affected by other environmental factors. Thirdly, the attenuation and dissipation of the space charge measurement may bring certain amount of errors.

Based on the calculated electric field distribution, the distortion factor of the electric field $\Delta \mathrm{E}$ can be calculated by the equation:

$$
\Delta \mathrm{E}=\frac{E_{\max }-E_{a v}}{E_{a v}} \times 100 \%
$$

where the $E_{\max }$ is the maximum value of the electric stress in the dielectric bulk due to the space charge accumulation; $E_{a v}$ is the average value of the applied electric stress. The electric field enhancement for each sample is illustrated in Fig. 10.

The field enhancements in the fresh oil samples are about $40 \%$; while more than $100 \%$ in the aged oil samples. Compared with the results without oil film obtained in our previous study [10], the field enhancement is much larger for each type of samples (about 15\% in the fresh oil samples; $45 \%$ in the aged oil samples).

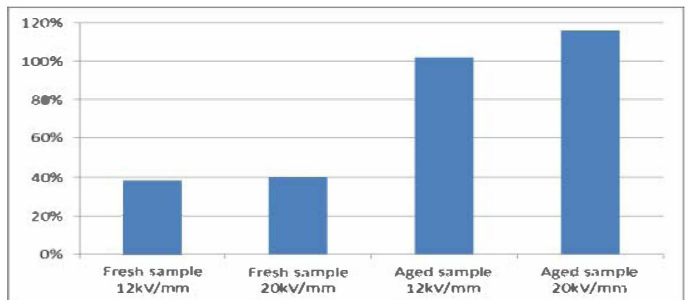

Fig. 9. Electric field enhancements for different samples.

\section{CONCLUSION}

In this study, the space charge dynamics in the impregnated pressboard combined with oil film was investigated by the means of PEA technique. The results show that an interfacial charge peak is quickly formed upon the external voltage application. The different status of the oil can significantly influence the relaxation time constant and the "barrier" effect of the interface. In the aged oil samples, the negative charges can quickly pass through the interface and move into the pressboard, leading to a significant electric field enhancement in the middle of the pressboard in the combined system.

\section{ACKNOWLEDGMENT}

The authors are grateful to the National Grid for their financial support.

\section{REFERENCES}

[1] X. Wu, G. Chen, A. E. Davies, Y. Tanaka, S. J. Sutton and S. G Swingier, "Space charge measurements in polyethylene under DC and AC operating conditions using the PEA technique," Dielectric Materials, Measurements and Applications, pp. 57-62, 2000.

[2] M. Jeroense and P. Morshuis, "Space charge measurements on impregnated paper: a review of the PEA method and a disscussion of results," IEEE Electrical Insulation Magazine, vol. 13, no. 3, pp. 26-35, 1997.

[3] R. Ciobanu, I. Prisecaru and C. Schreiner, "Space charge evolution in thermally aged celluloe materials," in ICSD, Taulorw, France, 2004..

[4] Y. Zhou, Y. Wang, G. Li, N. Wang, Y. Liu, B. Li, P. Li and H. Cheng, "Space charge phenomena in oil-paper insulation materials under high voltage direct current," Journal of Electrostatics, vol. 67, pp. 417-421, 2009 .

[5] C. Tang, G. Chen, M. Fu and R. Liao, "Space charge behaviour in multilayer oil-paper insulation under different DC voltages and temperatures," IEEE Trans. Dielectr. Electr. Insul., vol. 17, no. 3, pp. 778-788, 2010

[6] J. Hao, G. Chen, R. Liao, L. Yang and C. Tang, "Infulence of moisture on space charge dynamics in multilayer oil-paper insulation," IEEE Trans. Dielectr. Electr. Insul., vol. 19, no. 4, pp. 1456-1464, 2012.

[7] M. Huang, Y. Zhou, Q. Sun, Y. Sha and L. Zhang, "Effect of interface on space charge behavior in multi-layer oil-paper insulation," in CEIDP, Montreal, QC, 2012

[8] H. Wang, W. Kai, Q. Zhu, Y. Cheng and S. Li, "The research on space charge property in the sample with two layers of oil-immersed-paper and oil," in ISH 2013, Seoul, Korea, 2013.

[9] Y. Li, Y. Yasuda and T. Takada, "Pulsed electroacustic method for measurement of charge accumulation in solid dielectrics," IEEE Trans DEI, vol. 1, no. 2, pp. 188-195, 1994.

[10] M. Hao, Y. Zhou, G. Chen, G. Wilson and P. Jarman, "Space charge behaviour in thick oil-impregnated pressboard under HVDC stresses," Solid Dielectrics (ICSD), 2013 IEEE International Conference on , 2013.

[11] Z. Xu and G. Chen, "Interfacial characteristics of space charge in multilayer LDPE," in $C M D$, Beijing, 2008. 